\title{
Objetivos priorizados del eje de lectura en la región del Maule, Chile: problemáticas y desafíos
}

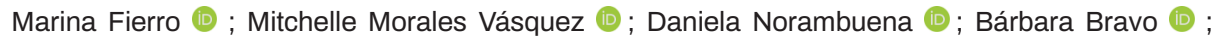 \\ Priscilla Contreras $\mathbb{C}$ \\ Universidad Católica del Maule (UCM), Chile
}

Resumen. Esta investigación se enmarca en el contexto educativo pandémico causado por la COVID-19. El objetivo de este trabajo fue analizar la implementación que realizan los docentes de Lengua y Literatura de los objetivos priorizados en torno al eje de lectura en $7^{\circ}$ y $/$ u $8^{\circ}$ Básico, en la Región del Maule, durante el contexto COVID-19. Se adoptó un enfoque mixto, en el que se revisaron los documentos Priorización Curricular y Didácticas para la proximidad, además, se implementó una encuesta online dirigida a docentes de diferentes comunas de la Región. Los resultados indicaron, por una parte, que los documentos concuerdan con la teoría, pero su categorización podría ser replanteada. Por otra parte, la encuesta reveló que los docentes han podido implementar los objetivos priorizados, pero existen dificultades atribuidas al contexto rural de la Región. Se concluye primero que existe debilidad respecto al Conocimiento Técnico Pedagógico del Contenido (TPACK), lo que abriría nuevas líneas de investigación. Segundo, lo planteado en la Priorización Curricular sobre los objetivos priorizados podría provocar un proceso de replanteamiento del Currículo establecido en Chile previo a la pandemia.

Palabras clave: COVID-19; priorización curricular; didácticas para la proximidad; TPACK; eje de lectura. Objetivos priorizados do eixo de leitura na região de Maule, Chile: problemas e desafios Resumo. Esta pesquisa insere-se no contexto educacional provocado pela pandemia COVID-19. Teve o fim de analisar a implementação dos objetivos de aprendizagem priorizados pelos professores de Língua e Literatura em torno do eixo de leitura no $7^{\circ} \mathrm{e} / \mathrm{ou} 8^{\circ}$ ano, na Região do Maule, durante o contexto da COVID-19. Adotou-se uma abordagem mista, na qual se revisaram os documentos Priorización Curricular e Didácticas para la proximidad. Além disso, implementou-se uma pesquisa on-line dirigida a professores de diferentes comunas da Região. Os resultados indicaram, por um lado, que os documentos concordam com a teoria, mas sua categorização poderia ser reconsiderada. Por outro lado, a pesquisa revelou que os professores conseguiram implementar os objetivos priorizados, mas existem dificuldades atribuídas ao contexto rural da Região. Primeiro, conclui-se que existe um ponto fraco no que diz respeito ao Conhecimento Técnico de Conteúdo Pedagógico (TPACK), o que abre novas linhas de pesquisa. Em segundo lugar, o que é proposto na Priorização Curricular sobre os objetivos priorizados poderia provocar um processo para repensar o Currículo estabelecido no Chile antes da pandemia.

Palavras -chave: COVID-19; priorização curricular; didáticas da proximidade; TPACK; eixo de leitura.

Implementation of the prioritized objectives of the reading in the Maule region, Chile: problems and challenges

Abstract. The scenario of this investigation is the pandemic educational context caused by COVID-19. The aim of this thesis was to analyze the implementation made by Language and Literature teachers of the prioritized objectives around the axis of reading in 7th and/or 8th grade, in the Maule Region, during the COVID-19 context. The research was carried out through a mixed research method, in which we reviewed the documents published by MINEDUC, Curricular Prioritization, and Didactics for Proximity. Also, an online survey was implemented, aimed at teachers from different communes in the region. The results indicated, first, that the documents agree with the theory, but the categorization should be reconsidered. Second, the survey revealed that teachers have been able to implement the prioritized objectives, but there are difficulties attributed to the rural context of the region. In conclusion, first, there is a weakness concerning the Technological Pedagogical Content Knowledge TPACK, which opens new lines of research. Second, what was raised in the Curricular Prioritization on the prioritized objectives could provoke a process of rethinking the Curriculum established in Chile prior to the pandemic. Keywords: COVID-19; curricular prioritization; didactics for proximity; TPACK; reading axis. 


\section{Introducción}

A partir del año 2020 las clases a nivel mundial sufrieron un cambio en su modalidad, se mutó hacia la educación a distancia, lo que requirió de mayores recursos y herramientas -como son la conexión a internet, aplicaciones, programas, computadores, celulares, entre otras- to que en diversos países fue complejo, ya que no se contaba con debido acceso. Además, surgió un cuestionamiento a la forma tradicional del proceso de enseñanza-aprendizaje, demostrando que requiere una actualización y adaptación a un contexto sin precedentes. En Chile, como medida para dar continuidad al proceso enseñanza-aprendizaje, desde el Ministerio de Educación (MINEDUC) se elaboró y presentó la Priorización Curricular (MINEDUC, 2020c), que consiste en una selección de objetivos de aprendizaje (OA) para las diferentes asignaturas y niveles académicos.

Entre las medidas que ha tomado el país ante la pandemia, las que también son acatadas y utilizadas por gran parte de la Región del Maule, surgieron iniciativas como TV Educa, Aprendo en casa, la plataforma Aprendo en línea, entre otras. Estas han funcionado como medios para llevar la educación hasta el hogar de los estudiantes. Sin embargo, los planes de emergencia han ido más allá, pues, en vista de las características del proceso, se formó una Mesa Social COVID-19 que colaboró con el MINEDUC. Dado lo anterior, se determinó que el currículo y las formas en las que se llevaban los procesos educativos debían adaptarse al contexto, por ello, se publicaron dos documentos claves Priorización Curricular (2020c) y Didácticas para la Proximidad (2020). En estos documentos se incluyeron los apartados de Lengua y Literatura que abordan la habilidad de lectura, la que es claramente transversal a todas las asignaturas, ya que es clave en el aprendizaje y prioritaria para el ingreso a la educación superior, al mismo tiempo, es uno de los puntos críticos de la educación en Chile.

Desde esta perspectiva Moreno (2020) y Cotino (2020) plantean que la situación de la educación es delicada y expone ejemplos de vulnerabilidad en varios países, pero también trae una serie de oportunidades en cuanto a modalidades de trabajo y la actualización del proceso de enseñanza y aprendizaje. En el caso específico de Cotino (2020) plantea que, a través de la aplicación de procesos, conocimientos y metodologías del área pedagógica, como son la transposición didáctica, el conocimiento del uso de tecnologías en torno a la disciplina (TPACK) y la aplicación del Flipped classroom, la modalidad virtual podía ser viable y efectiva.

Debido a lo anterior, la presente investigación consiste en un estudio mixto en el que se analiza la implementación que realizan los docentes de Lengua y Literatura de los objetivos priorizados en torno al eje de lectura en $7^{\circ}$ y $/$ u $8^{\circ}$ Básico, en la Región del Maule, durante el contexto COVID-19. A partir de este propósito se plantean cuatro objetivos específicos. El primero, analizar lo que estipula la Priorización Curricular de Lengua y Literatura en torno al eje de lectura. El segundo, examinar las orientaciones que ha entregado el Ministerio de Educación para implementar la Priorización Curricular en el contexto de la modalidad a distancia durante la pandemia provocada por la COVID-19. El tercero, distinguir las características del proceso educativo realizado por los docentes de la asignatura lengua y literatura en el contexto COVID-19. Por 
último, contrastar las experiencias de docentes de la asignatura Lengua y Literatura, que cuenten con conectividad con aquellos que no la poseen, en la implementación de la Priorización Curricular.

La pregunta de investigación es: ¿Cómo implementan los docentes de la asignatura Lengua y Literatura, los objetivos priorizados del eje de lectura propuestos por el MINEDUC (2020c), en séptimo y/u octavo Básico de la Región del Maule durante en el contexto COVID-19?

Mientras que la hipótesis plantea que los docentes de la asignatura Lengua y Literatura, implementan los objetivos priorizados del eje de lectura propuestos por el MINEDUC (2020c), en séptimo y octavo básico, en la región del Maule, durante la pandemia provocada por la COVID-19, de acuerdo con el contexto del procesoenseñanza aprendizaje, por lo que varían aspectos como la selección de textos, su extensión y los medios de entrega.

\section{Chile en el contexto latinoamericano}

En América Latina, según un informe realizado por la CEPAL y la UNESCO (2020), 160 millones de estudiantes fueron afectados por la suspensión de las clases presenciales. Mientras que el Banco Mundial (2020) afirma que el 60\% de las personas en Latinoamérica no utilizan internet, sobre todo en zonas rurales o de alta pobreza. Por su parte, el director internacional del Instituto de Educación Superior de la UNESCO, Pedró (2020, p. 3) indica que "en América Latina, solo el 52\% de los hogares cuenta con equipamiento tecnológico y conectividad de banda ancha".

Con tales antecedentes, se evidencia que Latinoamérica no estaba preparada para el cambio drástico de la modalidad de enseñanza. Dado lo anterior, los países tuvieron que adecuar el currículum y generar espacios y medios accesibles para los estudiantes. Es así como el BID (2020, p. 6) expresa que esta nueva modalidad cuenta "con una combinación de soluciones y medios de primera generación (materiales impresos, radio y televisión) y de segunda generación (plataformas, sistemas de gestión de aprendizajes) para entregar contenido y mantener algún nivel de interacción entre escuelas y estudiantes".

En lo que respecta a las medidas tomadas por los países latinoamericanos durante la pandemia, la CEPAL y la UNESCO (2020) señalan que 29 implementaron modalidades a distancia; de estos, 26 recurrieron al internet, 24 diseñaron estrategias educativas sin conexión a internet y 22 trabajan con modalidades sincrónicas y asincrónicas. Destacan entre los 29 países, cuatro -Bahamas, Costa Rica, Panamá y Ecuador- que se encuentran netamente con clases online en vivo. Además de los 23 países -Chile, Perú y México, entre otros- que se encuentran transmitiendo por radio o televisión programas que apoyan el desarrollo del aprendizaje. Cabe señalar, el Sistema de Información de Tendencias Educativas en América Latina (SITEAL) (2020) logró conocer la situación de diferentes naciones de América Latina entre las cuales solo tres indican haber realizado un ajuste en su currículo ante la pandemia, estos son Panamá, Ecuador y Chile.

En América Latina y el Caribe la población rural, según indica la OIT (2020), es de 123 millones aproximadamente. En países como Bolivia, Colombia, Perú y Argentina, las tasas de ruralidad llegan a 30,2\%, 18,9\%,21,9\% y $8 \%$, respectivamente (Banco 
Mundial, 2019). Mientras que, en Chile, corresponde a un 12,2\% de la población. En el caso específico de la Región del Maule, cuenta con una de las mayores tasas de ruralidad del país, con un 26,8\% (INE, 2017). Lo anterior es importante, ya que según lo mencionado anteriormente por el Banco Mundial (2020), es en las zonas rurales donde se presentan mayores dificultades de conectividad, por lo que la realidad educativa de dicha región, puede ser similar a la de los países anteriormente mencionados.

A esto se suma que la región cuenta con la tasa de analfabetismo más alta en la población de 15 años o más con un porcentaje de 6,2 (Ministerio de Desarrollo social, s.f.), según la encuesta Casen del 2015. Otro dato relevante, es que la región ha tenido un alza en su población extranjera, el 2014 (Micheletti, 2019) vivían 5.021 extranjeros en la región. Durante el 2017 este número aumentó a 10.780. Dentro de ese mismo año, se tiene registro (Morales et al., 2019) de que en la séptima región había 562 alumnos extranjeros matriculados en el sistema escolar, esta cifra ascendió a 3.176 estudiantes el año 2019 (MINEDUC, 2020a).

\section{Justificación}

Las condiciones de la Región del Maule son precarias para enfrentarse al cambio en la modalidad educativa, no solo existe una falta de recursos esenciales como el acceso a internet o un equipo, sino que también hay un alto índice de ruralidad y una situación alarmante en el nivel de alfabetización. Por tanto, es de suma importancia monitorear cómo se realiza la implementación los objetivos priorizados en el eje de lectura, pues es una de las habilidades que contempla la alfabetización y es esencial tanto para el desarrollo académico como recreativo.

Se debe agregar que las características que presenta la mencionada región, pueden ser la representación de las condiciones que se dan en diferentes ciudades y comunas que no sean las capitales de diferentes países de Latinoamérica. A esto se suma el hecho de que Chile, al igual que muchos otros países latinoamericanos, toma como referencia los resultados y las posteriores observaciones de la prueba estandarizada del Programa Internacional de Evaluación de los Alumnos (PISA). Entonces, al profundizar en el caso de la región, no solo se verían los resultados de la implementación de las diversas medidas tomadas por el MINEDUC, sino que también serviría como proyección y punto comparativo para el resto de los lugares que tengan condiciones similares en el continente latinoamericano.

\section{Efectos de la COVID-19 en la educación chilena}

En educación, debido a la suspensión de clases, se identifican dos problemas. El primero es la sobrecarga laboral, pues los profesores deben preparar mayor cantidad de material, por lo que "la escuela está en la casa [...] las jornadas de trabajo no tienen límites" (Bareiro, 2020, p. 81). El segundo es la pérdida de aprendizajes, que según el MINEDUC (2020b) podría ser estimada en un promedio de $88 \%$ que varía de acuerdo a los recursos económicos de los estudiantes. Dado lo anterior, Sandra Moreno (2020) destaca los conceptos Transposición Didáctica y TPACK, como fundamentales para la adaptación al nuevo contexto educativo. El primer concepto es definido por Yves Chevallard (1997) como la transformación de conocimientos disciplinarios, para que sean comprendidos por quienes no saben de estos (citado en 
De Faria, 2006). Mientras que el segundo consiste en la aplicación de la didáctica en el área disciplinar, en que se utilicen diversas tecnologías acordes con los objetivos de aprendizaje y las características del grupo curso (Mishra y Koehler, 2009). Por tanto, la conjugación de ambos permitirá un desarrollo adecuado para las clases con modalidades a distancia.

\section{Modalidades de enseñanza a distancia}

En cuanto a modelos pedagógicos de enseñanza, las modalidades online han sido fundamentales, entre ellas se reconocen el Blended Learning (B-Learning), Flipped classroom y E-learning. Pese a la existencia de las modalidades mencionadas, en Chile se han categorizado bajo otros nombres que corresponden a sincrónicas y asincrónicas. La primera consiste en actividades en que los participantes realizan una acción simultánea, como una video llamada (Propuestas Educación Mesa Social COVID-19, 2020). La segunda es completamente diferente, puesto que consiste en "actividades en que los participantes no coinciden en el mismo tiempo, por ejemplo, la observación de un video o la resolución de una guía" (Propuestas Educación Mesa Social COVID-19, 2020, p. 16). Las dos modalidades mencionadas anteriormente, surgieron como una opción para mantener activo el proceso educativo, y se consideren aquellos estudiantes con conectividad y sin ella.

\section{Priorización curricular y didácticas para la proximidad}

Las Bases Curriculares se definen como flexibles y buscan construir los lineamientos para que el aprendizaje sea continuo y acumulativo, para ello, se divide en asignaturas que presentan Objetivos de Aprendizaje (OA). El diseño curricular flexible implica seleccionar, organizar y distribuir los contenidos, lo que debe hacerse en consideración de la pertinencia, utilidad social e individual, contextualización, aplicabilidad e impacto (Escalona, 2008). Los programas de estudio son desarrollados a partir de lo estipulado en las Bases curriculares, según Henríquez (2019, p. 20) "los Programas de Estudio elaborados por el MINEDUC integran el uso de las Tecnologías de la Información y la Comunicación (TIC) en todas las asignaturas con el propósito de utilizar estrategias de búsqueda y selección de la información que sea relevante y confiable". La asignatura Lengua y Literatura tiene dos enfoques, cultural y comunicativo, y está dividida en cuatro ejes; lectura, escritura, comunicación oral e investigación. El eje de lectura pretende formar lectores que gocen de la lectura y que recurran a ella para cumplir con diferentes propósitos. En $7^{\circ}$ Básico se proponen 11 OA relacionados con el eje anteriormente mencionado, mientras que en $8^{\circ}$ Básico se establecen 12 OA. La Priorización Curricular nace a partir de la necesidad de flexibilización de las bases curriculares para cada nivel educativo, se realiza una selección de OA distribuidos en dos niveles ( 1 y 2 ). En $7^{\circ}$ y $8^{\circ}$ Básico, en el eje de lectura, en el nivel 1 se priorizaron 2 OA, mientras que en el nivel 2 se seleccionó 1 OA. Para poder trabajar la priorización curricular, que se debe mantener aproximadamente hasta fines del 2021, se redactaron las Didácticas para la Proximidad que propone orientaciones en cuanto a actividades, tiempo, contenidos, entre otros. 


\section{Lectura}

La lectura, es entendida como un proceso interactivo entre el lector y el texto, además de ser una herramienta cultural que permite entender a los otros y a sí mismos (Núñez, s.f.). Para abordar la lectura en clases, es fundamental la aplicación de estrategias sistematizadas, estas pueden ser planteadas en función de la comprensión y en consideración de los niveles que plantean las taxonomías de Thomas Barret en 1968 (Pérez, Bermúdez y Gallego, 2017) y Robert J. Marzano en 2001 (Gallardo, 2009). Según Núñez (sf), en clases presenciales se pueden aplicar las siguientes estrategias: predicción y verificación, lectura rápida o panorámica, explicitación de objetivos, procesamiento del vocabulario, autopreguntas, síntesis, conexiones, inferencias, visualización, planificación, monitorización, supervisión y evaluación.

\section{Estrategias de lectura en modalidades online}

En modalidades online la recomendación es trabajar las mismas estrategias que se utilizan en la presencialidad, pero de manera explícita y con el uso complementario de herramientas TIC. La investigadora Nicole Brun-Mercer y el sitio Edutopia coinciden en estrategias para modalidades virtuales, pero Edutopia profundiza en el tema y sugiere establecer momentos sincrónicos que preparen a los estudiantes para enfrentarse a la lectura; entregar plataformas que sirvan para resolver o ejercitarse en vocabulario que sea desconocido; activar conocimientos previos, esto puede ser mediante actividades sostenidas en plataformas como: Google Forms, Google Open Gallery o encuestas, modelar la actividad, ya sea por cápsula o por sesión sincrónica, en que se utilicen estrategias de lectura como realizar autopreguntas, resumir, realizar diferentes tipos de lecturas o pensar en voz alta. La última estrategia también es sugerida por Coiro (2011), quien afirma que a través de un modelamiento pensando en voz alta, los estudiantes pueden identificar el procedimiento que realiza el docente, un experto al leer, y les permite reconocer y definir lo que pueden hacer en su lectura.

Otro aspecto relevante dentro de la modalidad online vinculado a la lectura, es que existe un cambio en el soporte de las lecturas al formato digital. Los textos en soporte electrónico según explica Carvalho et al. (2018) son y tienen un gran potencial para los docentes, pues entregan posibilidades de adaptación de los textos, realizar actividades interactivas, rápida localización de términos o conceptos dentro de los textos, entre otras. No obstante, todo el potencial tiene mayores posibilidades de ser efectivo con la integración del uso de las TIC en la educación. A esta perspectiva se suma la de los estudiantes, Carvalho establece que los estudiantes, a pesar de todas las utilidades y herramientas que presentan los libros digitales, los alumnos prefieren leer y estudiar por medio de libros en formato tradicional, pues perciben una mayor retención de los contenidos, mayores posibilidades de manipular el texto -al destacar o realizar anotaciones al margen-y menores posibilidades de distraerse en su lectura. Lo anterior se ve fundamentado por lo que explican Jabr (2013) y Majul (2014) (citados en Carvalho et al., 2018), pues la lectura digital al estar retenida en una pantalla, limita las capacidades de exploración de los lectores, tiende a requerir que deban ajustar el texto antes de leerlo y elimina el estímulo del tacto al pasar a la página, por lo que quien lee no genera una representación mental del escrito tan amplia como al leer en papel. 
Sin embargo, existen otras investigaciones sobre lectura digital en modalidad e-learning, en que se ha descubierto que el soporte no incide en la comprensión de lectura, sino que depende de las habilidades lectoras particulares de los sujetos (Flores, Díaz y Lagos, 2017). Además, las estrategias de aplicación digital mejoran significativamente la comprensión de textos narrativos, pero que aun en el aspecto inferencial faltaría ajustar, quizá con la incorporación de una actividad didáctica más reflexiva (Novoa, 2019). Mientras que en ambientes $b$-learning, se evidencia que la experiencia en ambientes virtuales amplia el panorama de lectura a los estudiantes y les provee diversos recursos que contribuyen a la comprensión integral de los textos abordados (Montoya, Gómez y García, 2016).

\section{Marco metodológico}

En esta investigación se trabajó con un enfoque metodológico mixto, es decir, una mezcla de los enfoques cualitativo y cuantitativo, debido a que permite tener una perspectiva más amplia del fenómeno estudiado. Además, la metodología posibilita el desarrollo de un modelo un tanto artesanal que se acomode a las necesidades de la investigación. En cuanto a las técnicas para recolectar información que se utilizaron, fueron dos. La primera, fue un contraste entre la Priorización Curricular de Lengua y Literatura (MINEDUC, 2020c) con lo que indican las Bases Curriculares y las taxonomías de Barret (1968) y Marzano (2001). Además, se comparó las orientaciones señaladas por Didácticas para la Proximidad (MINEDUC, 2020) con lo planteado por las estrategias de lecturas presentadas en el marco teórico y lo estipulado por las Bases Curriculares. La segunda, fue una encuesta de aplicación online a docentes de Lengua y Literatura a cargo de $7^{\circ}$ y/u $8^{\circ}$ Básico durante la modalidad a distancia en la Región del Maule y recabó información sobre aspectos como las condiciones laborales, implementación de la priorización, el eje de lectura y el uso de tecnologías; la muestra fue voluntaria y contó con la participación de 32 docentes de Lengua y Literatura. Cabe mencionar, en la actualidad no se cuenta con un registro actualizado de la cantidad de pedagogos de la disciplina que hay en la región, lo más cercano es lo que se indica en la Minuta Docentes 2019 que contabiliza un total de 16.678 docentes, sin diferenciar la disciplina o nivel a los que pertenecen (MINEDUC, 2019).

La investigación se enmarcó en el contexto provocado por la pandemia, específicamente, durante el segundo semestre del año académico 2020, que se caracterizó por el trabajo bajo la modalidad a distancia. En el caso específico de los encuestados, ellos son profesores de la Región del Maule de comunas urbanas y rurales, vinculados a establecimientos municipales, sostenidos por fundaciones o particulares.

\section{Resultados de la revisión de documentos y encuesta}

Respecto de los objetivos priorizados para el eje de lectura en $7^{\circ}$ y $8^{\circ}$ Básico, ambos cuentan con tres $\mathrm{OA}$, dos en nivel 1 que hacen referencia al análisis de textos literarios y no literarios, y uno en nivel 2 que aborda las estrategias de lectura. En general, los objetivos cumplen con lo que estipulan las Bases Curriculares y con lo que se plantea en las taxonomías de Barret (1968) y Marzano (2001) en cuanto al análisis y aplicación de estrategias, pero no cumplen con el aspecto estético que se establece en los documentos anteriormente mencionados. 
El informe de Didácticas para la Proximidad (MINEDUC, 2020), plantea que, respecto al eje señalado, el trabajo se debe enfocar en elaborar y plantear objetivos de lectura claros para los estudiantes; diseñar desafíos de lectura que motiven a los alumnos a trabajar con un texto; acompañar y modelar la lectura; plantear apoyo durante la lectura para motivar; procurar la selección de textos breves; crear espacios de diálogo participativo; promover la lectura personal por placer y fomentar el uso de recursos digitales para complementar la lectura.

Dentro de esta investigación se utilizó como recurso una encuesta a través de Google forms, estuvo disponible durante octubre y noviembre del 2020 y fue compartida a establecimientos de la Región del Maule; Constitución, Curepto, Empedrado, Maule, Pelarco, Pencahue, San Clemente, Cauquenes, Pelluhue, Vichuquén, Talca, Curicó, Hualañé, Licantén, Molina, Rauco, Romeral, Sagrada Familia, Teno, Linares, Yerbas Buenas, San Javier, Villa Alegre, Parral, Longaví, Colbún, Río Claro, San Rafael, Chanco y Retiro. Se obtuvieron las respuestas de 32 docentes de diferentes edades dentro del rango de 20 a 51 o más años, con años de experiencia en docencia que van desde 1 año a más de 31 años. En cuanto a tipos de establecimientos de los que provienen los profesores, destacaron los de instituciones municipales, pero también respondieron de fundaciones y particulares. Por último, respondieron docentes que se hacían cargo tanto de $7^{\circ}$ y $8^{\circ}$ Básico y también pedagogos a cargo de solo uno de ellos.

Figura 1. Mapa político administrativo de la Región del Maule, Chile
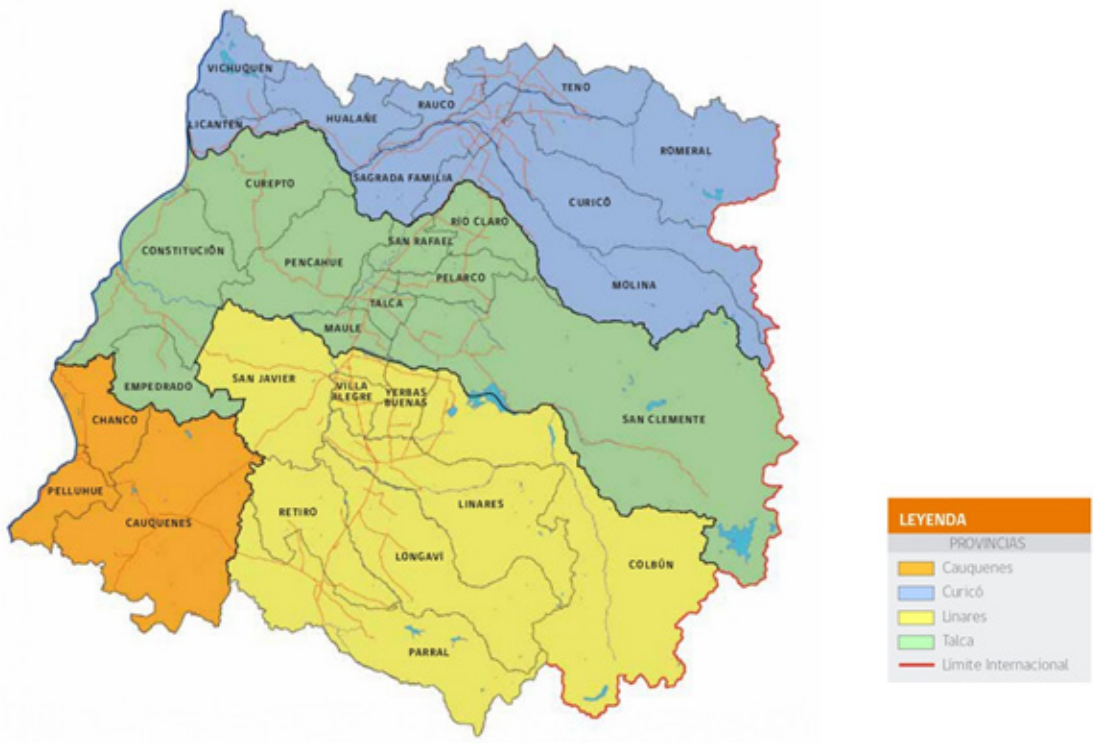

Nota. Adaptado de División político administrativa (p. 43), por Programa Zonas Rezagadas, VOA (2015),

Acontinuación, se presenta una selección de los resultados de cada sección de la encuesta. En la primera sección, sobre las condiciones laborales de los docentes, estos indican que estas han cambiado, puesto que la gran mayoría de profesores trabajan desde sus casas. Tanto para la modalidad sincrónica como asincrónica, pese 
a las circunstancias, han logrado mantener una comunicación fluida con los miembros de la comunidad educativa, aunque, la mayor parte de los profesores afirmó autofinanciar sus recursos para trabajar.

Gráfico 1. Mi trabajo pedagógico durante la enseñanza en contexto de pandemia ha sido en modalidad(es)

Enunciado 1: Mi trabajo pedagógico durante la enseñanza en contexto de pandemia ha sido en modalidad(es):

Entrega de material en turnos éticos.

Modalidad asincrónica (envío de capsulas, guías, presentaciones entre otros).

Modalidad sincrónica (reuniones con cierta frecuencia por plataforma)

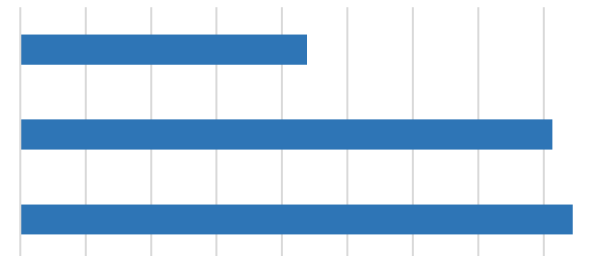

$\begin{array}{lllllllllllllllllll}0 \% & 10 \% & 20 \% & 30 \% & 40 \% & 50 \% & 60 \% & 70 \% & 80 \% & 90 \%\end{array}$

Fuente. Elaboración propia.

De acuerdo con el gráfico, un $84,4 \%$ de docentes trabaja con modalidad sincrónica. Mientras que un $81,3 \%$ trabaja con modalidad asincrónica. Finalmente, un $43,8 \%$ trabaja con entrega de material en turnos éticos, que consisten en períodos de tiempo, en que los establecimientos educacionales permanecen abiertos a la comunidad (MINEDUC, 2020d). Cabe señalar, este enunciado permitía seleccionar más de una opción, por lo que se demuestra que no se adoptó una sola modalidad para el desarrollo del proceso educativo, sino que se han complementado las opciones disponibles.

La segunda sección corresponde a los aspectos pedagógicos, se evidencia que la mayoría de los establecimientos trabajan según lo establecido en la Priorización Curricular, ya que consideran que los objetivos permiten abordar las diversas temáticas y contenidos de los cursos. Además, señalan que se implementan diversos tipos de materiales para motivar e interesar a los estudiantes.

Gráfico 2. Hubiera escogido otros objetivos de aprendizaje para la priorización curricular

Enunciado 2: Hubiera escogido otros objetivos de aprendizaje para

la priorización curricular

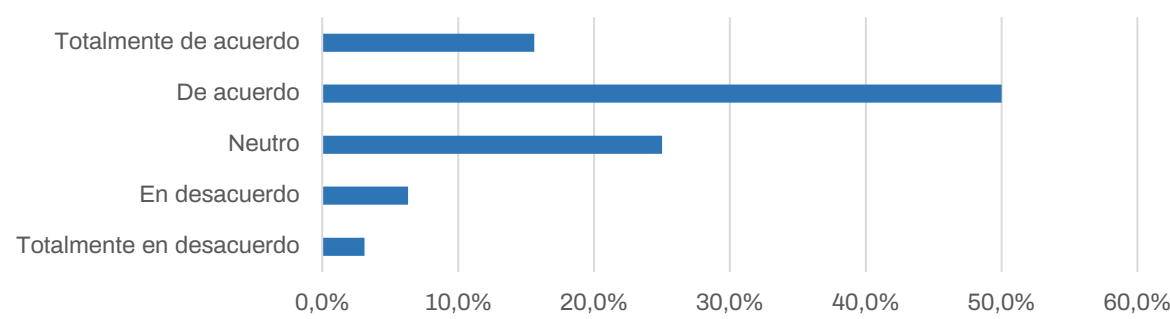

Fuente. Elaboración propia. 
Pese a que la mayoría indica estar conforme con los objetivos priorizados, el enunciado del Gráfico 2 demuestra que un 50\% está de acuerdo en que hubiera escogido otros OA para la priorización curricular. Mientras que un $25 \%$ se declara neutro. Un $15,7 \%$ está totalmente de acuerdo. Un 6,3\% está en desacuerdo. Finalmente, un $3,1 \%$ señala estar totalmente en desacuerdo.

Con lo que respecta a la sección relacionada con el eje de lectura, la mayoría afirmó que propone actividades motivantes para atraer a los estudiantes, dirigidas al análisis literario, enfocándose en el aprendizaje literario y el desarrollo de habilidades. Por lo que se infiere que existió una buena transposición didáctica.

Tabla 1. Resultados sección eje de lectura

\begin{tabular}{lccccc}
\hline \multicolumn{1}{c}{ Enunciado } & $\begin{array}{c}\text { Totalmente } \\
\text { en des- } \\
\text { acuerdo }\end{array}$ & $\begin{array}{c}\text { En des- } \\
\text { acuerdo }\end{array}$ & Neutro & $\begin{array}{c}\text { De } \\
\text { acuerdo }\end{array}$ & $\begin{array}{c}\text { Totalmente } \\
\text { de acuerdo }\end{array}$ \\
\hline $\begin{array}{l}\text { 2. Diseño actividades variadas que } \\
\text { motivan a los estudiantes, y ade- } \\
\text { cuadas para generar aprendizajes } \\
\text { (guías de autoaprendizaje, lecturas } \\
\text { mensuales, envío de ppty cápsulas } \\
\text { explicativas, etc.) }\end{array}$ & $0 \%$ & $3,1 \%$ & $3,1 \%$ & $50 \%$ & $43,8 \%$ \\
$\begin{array}{l}\text { 3. Considero que he logrado rea- } \\
\text { lizar actividades que propician el } \\
\text { aprendizaje y el desarrollo de las }\end{array}$ & $0 \%$ & $3,1 \%$ & $9,4 \%$ & $50 \%$ & $37,5 \%$ \\
habilidades de lectura. & & & & & \\
$\begin{array}{l}\text { 4. Las actividades de lectura que } \\
\text { diseño están dirigidas al análisis, } \\
\text { tanto de textos literarios como no } \\
\text { literarios, de acuerdo a sus pro- } \\
\text { piedades y las habilidades de los } \\
\text { estudiantes. }\end{array}$ & $0 \%$ & $0 \%$ & $0 \%$ & $53 \%$ & $46,9 \%$ \\
$\begin{array}{l}\text { 5. Acompaño y modelo diferentes } \\
\text { etapas del proceso de lectura. }\end{array}$ & $3,1 \%$ & $6,3 \%$ & $6,3 \%$ & $53,1 \%$ & $31,3 \%$ \\
\hline
\end{tabular}

Fuente. Elaboración propia

El cuarto apartado enfocado en el uso de las TIC, tuvo un porcentaje mayoritario de docentes que afirmó no tener las competencias necesarias para lograr la modalidad online a cabalidad, mientras que otros señalaron la necesidad de capacitaciones para afrontar el escenario virtual o también el aprendizaje autónomo para llevarlo a cabo.

Tabla 2. Resultados sección TIC

\begin{tabular}{lccccc}
\hline \multicolumn{1}{c}{ Enunciado } & $\begin{array}{c}\text { Totalmente } \\
\text { en des- } \\
\text { acuerdo }\end{array}$ & $\begin{array}{c}\text { En des- } \\
\text { acuerdo }\end{array}$ & $\begin{array}{c}\text { Neutro } \\
\text { De }\end{array}$ & $\begin{array}{c}\text { Detalmente } \\
\text { acuer- } \\
\text { do }\end{array}$ & $\begin{array}{r}\text { To acuerdo } \\
\text { de }\end{array}$ \\
\hline $\begin{array}{l}\text { 1. Mis conocimientos tecnológicos } \\
\text { fueron suficientes para adaptarme } \\
\text { a la enseñanza no presencial. }\end{array}$ & $0 \%$ & $21,9 \%$ & $15,6 \%$ & $40,6 \%$ & $21,9 \%$ \\
$\begin{array}{l}\text { 2. He recibido por parte delestable- } \\
\text { cimiento capacitación tecnológica. }\end{array}$ & $6,3 \%$ & $18,8 \%$ & $28,1 \%$ & $31,3 \%$ & $15,6 \%$ \\
\end{tabular}




\begin{tabular}{lccccc}
\hline Enunciado & $\begin{array}{c}\text { Totalmente } \\
\text { en des- } \\
\text { acuerdo }\end{array}$ & $\begin{array}{c}\text { En des- } \\
\text { acuerdo }\end{array}$ & $\begin{array}{c}\text { Neutro } \\
\text { acuer- } \\
\text { do }\end{array}$ & $\begin{array}{c}\text { Totalmente } \\
\text { de acuerdo }\end{array}$ \\
\hline $\begin{array}{l}\text { 3. He aprendido autónomamente } \\
\text { sobre diversas herramientas que } \\
\text { me permitan optimizar el proceso } \\
\text { enseñanza aprendizaje. }\end{array}$ & $0 \%$ & $3,1 \%$ & $3,1 \%$ & $34,4 \%$ & $59,4 \%$ \\
\hline
\end{tabular}

Fuente. Elaboración propia

Por último, en la sección de reflexión, los docentes indicaron que las principales dificultades se relacionan con la nueva modalidad a distancia, ello implica el uso del tiempo, evaluaciones, uso de tecnologías, la falta de recursos y conexión por parte de los estudiantes.

Tabla 3. Síntesis de los resultados sección Reflexión

\begin{abstract}
De acuerdo con su experiencia de enseñanza durante el año 2020, ¿cuáles han sido sus principales dificultades para implementar los objetivos priorizados del eje de lectura en $7^{\circ}$ y/o $8^{\circ}$ Básico?

Puede referirse al uso de las TIC, la adaptación de sus planificaciones a la Priorización Curricular, construcción de instrumentos evaluativos, tiempos de implementación, modalidades educativas, conocimientos de los estudiantes, etc.
\end{abstract}

La principal dificultad ha sido el cambio de modalidad y la adaptación a esta.

La principal dificultad ha sido que los estudiantes no tengan acceso a conexión a internet y otros recursos como impresora o computador.

La principal dificultad está en el manejo de los nuevos programas y aplicaciones tanto para docentes como para estudiantes.

La principal dificultad ha sido la elaboración de materiales y evaluaciones.

La principal dificultad es no poder saber cómo van en sus aprendizajes, por la imposibilidad de monitorear.

La principal dificultad ha sido el fomento del hábito lector.

La principal dificultad ha sido los tiempos para implementar y estar con los estudiantes

La principal dificultad es la desmotivación de los estudiantes.

\section{Fuente. Elaboración propia.}

Las respuestas de esta sección dan cuenta sobre la perspectiva que tienen los profesores frente a lo que ha obstaculizado su trabajo y apuntan principalmente a la modalidad y los problemas de conexión de los estudiantes como las grandes barreras a las que se han enfrentado dentro de este contexto. Además, se evidencian dificultades en la adaptación en otros temas como son el manejo de tiempos y la construcción de materiales, lo último podría potenciarse con el bajo dominio de las nuevas tecnologías que se manifiesta tienen tanto los profesores y los estudiantes. Por tanto, los impedimentos no estarían asociados a variables de la disciplina.

Otros temas que no fueron recurrentes en las respuestas son la alusión a las limitaciones del Currículo Priorizado, problemas de gestión por parte del establecimiento, los conocimientos y habilidades previas de los estudiantes y la falta de motivación. 


\section{Discusión}

Dentro de la revisión de los objetivos priorizados de $7^{\circ}$ y $8^{\circ}$ Básico, podemos afirmar que cumplen con lo que se establece en las Bases Curriculares en el punto en que abordan tanto textos literarios como no literarios. A ello se suma que también abordan las estrategias de lectura, pero lo dejan en un segundo lugar de prioridades, lo que podría ser replanteado si se ve desde la perspectiva de las modalidades online, que requieren de una mayor autonomía. Por tanto, sería apropiado propiciar la sistematización de estrategias antes de trabajar en el análisis propiamente tal, de esta manera se podría proyectar el análisis como un trabajo a futuro y más independiente. Por último, los objetivos no cumplirían con el desarrollo de un gusto estético ni con formar criterios de selección de textos, esto puede ser por no tomar en cuenta criterios planteados desde las taxonomías de Barret (1968) y Marzano (2001). Los últimos aspectos de cierta manera se contradicen con lo señalado en Didácticas para la Proximidad, en que aseguran que la lectura puede servir como "una puerta de escape" por su efecto estético y presentación de mundos diferentes.

En cuanto a las orientaciones entregadas por el documento Didácticas para la Proximidad en el eje de lectura, se aprecia concordancia a grandes rasgos con lo que se plantea desde la teoría sobre la lectura, sus estrategias y estrategias sugeridas para el ambiente online. Sin embargo, falta profundizar más en el cómo, pues como se evidencia en los resultados de la encuesta, los docentes carecían de la preparación tecnológica requerida para esta instancia y desconocen herramientas que pueden utilizar para aplicar las sugerencias. En consecuencia, parece conveniente recurrir a las recomendaciones en aplicaciones por parte de la fundación Edutopia, las que fueron presentadas en el marco teórico, o construir algo similar que sea congruente con la realidad de los pedagogos y sus estudiantes. Además, sería adecuado plantear los momentos definidos para cada actividad sugerida, de forma explícita, si lo pensamos desde lo que plantea Ferguson (2020), Brun-Mercer (2019) y Coiro (2011).

Entre nuestros objetivos para esta investigación figuraba realizar un contraste entre docentes que no presentaran las condiciones para realizar clases en modalidad sincrónica y los docentes que sí tenían esa posibilidad. Nuestras expectativas eran que las realidades y desarrollos de los procesos fueran completamente opuestos. No obstante, al examinar la realidad del $12,5 \%$ de los encuestados que no puede realizar clases sincrónicas, debido a la falta de conectividad, resulta ser que no se diferencia mucho de los docentes que sí están realizando clases, pues también en su mayoría utilizan más de 6 horas para realizar sus labores, manifiestan problemas por la falta de conectividad y el poco conocimiento del uso de tecnologías. Es necesario destacar que, dentro de estos encuestados, el $75 \%$ asegura mantener comunicación fluida con docentes, directivos, alumnos y apoderados, y que sienten que su establecimiento ha reaccionado bien ante la situación. Lo que nos hace pensar que las diferencias mínimas entre estos dos tipos de docentes pueden deberse a una adecuada transposición didáctica por parte de los establecimientos y los pedagogos, pues han tomado su contexto y realidad para transformar la forma en la que guían el proceso de aprendizaje. Mientras que los establecimientos educativos que sí contaban con conexión a internet, probablemente, su interés fue continuar realizando clases del mismo modo que en la modalidad presencial, por lo que su percepción del año académico 2020 
sea negativa, ya que no han podido abordar la misma cantidad de contenidos. Cabe señalar, la teoría presentada y las investigaciones realizadas en contextos virtuales, indica que es posible abordar la lectura exitosamente en modalidades de enseñanza a distancia, la diferencia es que es necesario que todo lo que se realice debe ser mencionado de manera explícita, para que los estudiantes sepan lo que están realizando y de qué manera les sirve. En el caso de los alumnos con clases asincrónicas o que han debido aprender mediante la realización de guías, es importante diseñar material en que se modele lo que deben hacer, con el fin de que los estudiantes puedan ver cómo se hace, para que luego ellos lo imiten.

Otro de nuestros objetivos era distinguir las características del proceso educativo realizado por los docentes de la asignatura Lengua y Literatura en el contexto COVID-19. Dado lo anterior, realizamos una encuesta que se refería a aspectos como las condiciones laborales, lo pedagógico, el eje de lectura, TIC y una reflexión sobre las principales dificultades de la implementación de los objetivos priorizados en $7^{\circ} \mathrm{y} / \mathrm{u}$ $8^{\circ}$ Básico. A partir de los resultados, tanto los profesores con conexión como los sin conexión, manifiestan que sus tareas relacionadas con la disciplina fueron posibles de realizar. No obstante, aunque realizaron diversas actividades, algunos manifiestan que ha sido difícil llevar a cabo los procesos de comprensión lectora, puesto que no todos los alumnos cuentan con los medios para imprimir y poder tomar notas o destacar, lo que afecta la comprensión, pues consideran que el material impreso es el óptimo para leer. Ejemplo de lo anterior es el caso de un docente (2020, s.p.) que indica: "[...] no todos los estudiantes tienen acceso a una impresora y en el caso de lenguaje es esencial tener material tangible donde puedan subrayar, destacar, tomar notas al margen, etc.". Esta idea es incompatible con lo señalado por las investigaciones presentadas, porque se indica que no hay grandes diferencias de comprensión entre la lectura digital y la lectura en material impreso, incluso, se señala que se pueden aplicar las mismas estrategias en los medios digitales como impresos. Cabe mencionar que, las herramientas virtuales de lectura si permiten añadir comentarios y destacar ideas importantes, lo que podría significar que los docentes carecen de conocimientos sobre la lectura digital, por lo que es necesario que sean capacitados en este ámbito, no tan solo por la modalidad de enseñanza provisoria que se ha adoptado, sino porque la sociedad exige la lectura en medios digitales, en la que es necesario conocer las herramientas disponibles.

Respecto a lo que manifiestan los encuestados dentro de la última sección de la encuesta sobre las dificultades, el foco está en el cambio de modalidad, la falta de recursos por parte de los estudiantes y el poco conocimiento en torno a las nuevas tecnologías. El primero de los problemas, sobre la modalidad, es peculiar, pues la modalidad sincrónica no es una metodología de enseñanza propiamente tal y desde el MINEDUC solo se especifica en que se lleva a cabo a través de una aplicación de videollamada y que idealmente debe mantenerse por menos de una hora. Por ello, pensamos que el problema puede responder a que la modalidad no tiene una estructura definida, como si la tienen las modalidades concebidas o compatibles con plataformas online (e-learning, b-learning y flipped classroom), y que esto podría ser parte de la solución. En la segunda dificultad debemos decir que responde por completo a los antecedentes y características establecidas dentro del capítulo 1, el acceso a inter- 
net y recursos tecnológicos se dificulta para la población chilena y en especial en la región del Maule. Por último, lo que se señala sobre la tecnología, consideramos que se relaciona con el hecho de que tengan dificultades en la construcción de materiales para sus clases usando aplicaciones y que esto como un todo, se debe a que los docentes no presentan TPACK, es decir, su dominio de la disciplina no tiene integrado el dominio y uso de la tecnología de forma funcional y aplicada con su especialidad.

Un aspecto interesante es que, pese a que los docentes señalan que han realizado lo que establece tanto la Priorización Curricular como las orientaciones entregadas en los lineamientos de Didácticas para la proximidad, su apreciación de lo realizado durante el año no sea satisfactoria. Pensamos que esto puede ocurrir, porque principalmente las sugerencias emitidas por el MINEDUC están pensadas para ambientes ideales en que estudiantes y profesores tienen una buena conexión a internet, poseen un computador personal para acceder a las clases, tienen impresoras para imprimir el material diseñado, entre otras. Sin embargo, la realidad no es esa, así que para llevar a cabo el proceso de enseñanza-aprendizaje, se han tenido que acomodar a la situación y buscar opciones para mantener el contacto con los estudiantes y/o apoderados, por lo que probablemente piensen que, si el contexto hubiera sido diferente, podrían haber obtenido mejores resultados.

Hay que mencionar además, que al analizar las preguntas de la encuesta que realizamos, los profesores sobre 41 años son quienes expresan que han tenido dificultades para seguir las orientaciones emanadas desde el MINEDUC y utilizar las TIC en su quehacer pedagógico, es importante indicar que este problema también lo presentan profesores más jóvenes, pero no con el grado de frecuencia que los mayores. Lo anterior permite interpretar que la edad no influye en los dominios tecnológicos que poseen los docentes, puesto que afecta a jóvenes como a mayores. También, es necesario recalcar que del total de los encuestados el 53,2\% considera que no ha recibido apoyo por parte del MINEDUC, lo que puede ser causado por una falta de información en cuanto a las charlas que ha transmitido la entidad por redes sociales o porque las herramientas y apoyos entregados por el Ministerio no responden a las necesidades de los profesores, lo que evidencia una falta de conocimiento en cuanto a las habilidades y dominio de la teoría de los educadores por parte del organismo.

Otro punto interesante para mencionar es que, pese a que la mitad de los docentes encuestados señalan estar conformes con los objetivos priorizados seleccionados, algunos de ellos declaran que hubieran escogido otros objetivos, de manera que, la percepción docente sobre esta selección de objetivos no es completamente satisfactoria. Sería bueno indagar más a fondo el porqué de esta postura, ya que como indican los fundamentos de la Priorización Curricular la selección fue realizada, debido a que los objetivos presentados son globales respecto de los que no fueron escogidos, por lo que, aunque no se presenten oficialmente, el desarrollo de los objetivos priorizados permitirá el abordaje de los demás. 


\section{Conclusiones}

A través del análisis y la discusión de los resultados obtenidos tanto de la revisión de documentos como la encuesta aplicada a docentes de Lengua y Literatura a cargo de $7^{\circ} \mathrm{y} / \mathrm{u} 8^{\circ}$ Básico de la Región del Maule que perseguían el propósito de analizar la implementación que realizan los docentes de Lengua y Literatura de los objetivos priorizados en torno al eje de lectura en $7^{\circ} \mathrm{y} / \mathrm{u} 8^{\circ}$ Básico, en la Región del Maule, durante el contexto COVID-19 y en consideración de la hipótesis. Se puede afirmar mediante la investigación que, en general, a pesar de presentar diversas dificultades asociadas a la pandemia y el acceso a recursos por parte de los estudiantes, los docentes de Lengua y Literatura a cargo de $7^{\circ}$ y/u $8^{\circ}$ Básico han logrado implementar los objetivos priorizados del eje de lectura realizando procedimientos congruentes a los planteados dentro de las orientaciones entregadas en Didácticas para la Proximidad.

De acuerdo con la revisión de los documentos, están en concordancia a grandes rasgos con la teoría, pero sería conveniente reconsiderar algunos puntos dentro de los textos, al mismo tiempo, que se actualice la información que entregan, pues como sabemos el mundo está en un cambio constante, por lo que los avances en metodología estrategias y otros aspectos aparecen de forma permanente. Dentro de estos cambios sería pertinente considerar la realidad de los docentes que aún no dominan el TPACK y desconocen herramientas que les pueden servir, sobre todo a los profesores sobre 41 años, pues ellos son quienes presentan menor conocimiento en esta área y para quienes, según lo manifestado en sus respuestas, el MINEDUC no ha sabido llevar la situación, ni les ha prestado apoyo. Con lo que respecta a los objetivos priorizados, reiteramos que apoyadas en la teoría y en la proyección de que esta situación puede extenderse, sería bueno priorizar la utilización de estrategias de lectura.

Por lo que se refiere a los resultados de la investigación, nos permitieron descubrir que existe la necesidad de capacitar tanto a estudiantes como docentes en el uso de nuevas tecnologías y las modalidades de aprendizaje, debido a que por una parte, se pensaba que los alumnos tenían conocimientos acerca del uso de la tecnología, pero los profesores señalan que al parecer el uso de las herramientas digitales se limita a las redes sociales, puesto que desconocen cómo enviar correos, usar Drive y/ o acceder a Google Meet, entre otros. Cabe señalar, aunque los programas de estudios estipulan el uso de las TIC, su propósito relacionado con las de estrategias de búsqueda y selección de información no es suficiente para el desarrollo del proceso enseñanza-aprendizaje, puesto que con las modalidades de clases (sincrónicas y asincrónicas) es más visible la debilidad en cuanto al uso de estas herramientas, ya sea en estudiantes como en profesores. Por otra parte, los educadores en su gran mayoría han tenido que aprender autónomamente y en paralelo a su desempeño en la labor de enseñanza, ya que el contexto les exigió adecuarse a la modalidad, lo que evidenció su debilidad en cuanto al TPACK.

Otro hallazgo realizado mediante la investigación es que las principales dificultades que experimentan los docentes son las características que tiene la región del Maule y las condiciones de vida de quienes la habitan, puesto que tal como se presentó en los antecedentes, dicha zona presenta el mayor porcentaje de ruralidad del país, por lo que existen grandes dificultades de conectividad, lo que perjudica el 
poder acceder a las clases sincrónicas, además, no todas las familias pueden proveer un computador, notebook, tablet o teléfono inteligente a los educandos para que estos puedan realizar las actividades lectivas.

Finalmente, es relevante destacar que la experiencia en la priorización de los objetivos desde el MINEDUC permite reflexionar sobre las posibilidades de reactualización de las Bases curriculares y la necesidad de una participación más democrática del profesorado chileno en la sistematización de las necesidades de la asignatura de Lengua y Literatura en distintos niveles de enseñanza. En consecuencia, la experiencia de la pandemia no solo revela carencias a nivel tecnológico y/o territorial, sino una desarticulación respecto de las necesidades de enseñanza en el eje de lectura que es transversal e impacta profundamente en el aprendizaje de diversas asignaturas.

Limitaciones de la investigación

- Complejidad del escenario que se atravesaba a nivel tanto mundial como nacional, que implicó depender de la calidad de la conexión y la adaptación de las metodologías de trabajo.

- Falta de investigaciones enfocadas en el impacto de emergencias dentro de la educación.

Recomendaciones para futuras investigaciones

- Investigar sobre la enseñanza del TPACK dentro de la formación docente.

- Investigar sobre el dominio de herramientas tecnológicas por parte de los profesores que están ejerciendo.

- Priorizar exploración de estrategias de lectura congruentes con la nueva modalidad para potenciar mayor autonomía del proceso de aprendizaje.

- Indagar sobre los aspectos que priorizarían los docentes.

\section{Referencias}

BID (2020). La educación en tiempos del coronavirus. Los sistemas educativos de América Latina y el Caribe ante COVID-19. Banco Interamericano de Desarrollo. Recuperado de https:// bit.ly/3fIfORm

Banco Mundial (2019). Población rural (\% de la población total) - Colombia, Bolivia, Perú, Argentina. Recuperado 25 de enero de 2021, de https://bit.ly/34K5M0p

Banco Mundial. (2020). La educación en América Latina enfrenta una crisis silenciosa, que con el tiempo se volverá estridente. Recuperado de https://bit.ly/3cenq0p

Bareiro, L. (2020). Ser docente en tiempos de pandemia: reflexiones para pensar el ejercicio docente en Paraguay. Kera Yvoty: reflexiones sobre la cuestión social, 5(número especial), 78-85. Recuperado de https://bit.ly/3vOxLle

Brun-Mercer, N. (2019). Online Teaching Strategies for the Classrooms. English Teaching Forum, 57(4), 2-11. Recuperado de https://bit.ly/3uQzN9w

Carvalho, J., Luengo, R., Casas, L. y Cubero, J. (2018). Para estudiar, ¿mejor el libro impreso o el libro digital?: un estudio exploratorio de naturaleza cualitativa. CIAIQ2018, 1, s.p. Recuperado de: https://bit.ly/2Ri6MWF

CEPAL y UNESCO (2020). La educación en tiempos de la pandemia de COVID-19. Recuperado de https://bit.ly/3hZeW19 
Coiro, J. (2011). Talking About Reading as Thinking: Modeling the Hidden Complexities of Online Reading Comprehension. Theory Into Practice, 50(2), 107-115. https://doi.org/10.1080/ $\underline{00405841.2011 .558435}$

Cotino, L. (2020) La enseñanza digital en serio y el derecho a la educación en tiempos del coronavirus. Revista de educación y derecho, 21, 1-29. Recuperado de https://bit.ly/3ia1KXf

De Faria, E. (2006). Transposición Didáctica: Definición, Epistemología, Objeto de estudio. Cuadernos de investigación y formación en educación matemática,2, s.p. Recuperado de https://bit. ly/3uMFy8i

Escalona, L. (2008). Flexibilidad curricular: elemento clave para mejorar la educación bibliotecológica. Investigación bibliotecológica, 22(44), 143-160. Recuperado de https://bit.ly/3pgaXi5

Ferguson, C. (2020). Adapting Reading Comprehension Instruction to Virtual. Edutopia: Learning. Recuperado de https://edut.to/3uG3sSQ

Flores, P., Díaz, A. y Lagos, I. (2017). Comprensión de textos en soporte digital e impreso y autorregulación del aprendizaje en grupos universitarios de estudiantes de educación. Revista Electrónica Educare, 21(1), 1-17. Recuperado de https://edut.to/3uG3sSQ

Gallardo, K. (2009). La Nueva Taxonomía de Marzano y Kendall: una alternativa para enriquecer el trabajo educativo desde su planeación. Recuperado de https://bit.ly/3zgDXuQf

Henríquez, A. (2019). Análisis comparativo del uso de las TIC en comprensión lectora en documentos curriculares de tres países Chile Colombia y México (Tesis de maestría). Recuperado de https://bit.ly/3ph06o9

INE (s.f.). WEB Diseminación CENSO 2017-Maule. Instituto Nacional de Estadísticas. Recuperado de https://bit.ly/3cbvBLe

Micheletti, S., Cubillos J., González C., y Valdés De La Fuente, E. (2019). Inserción laboral de migrantes en los territorios agrarios de Chile: el caso de la región del Maule. Cultura-hombre-sociedad, 29(1), 33-58. https://dx.doi.org/10.7770/0719-2789.2019.cuhso.02.a03

Ministerio de Desarrollo Social. (s.f.). Región del Maule. Casen 2015. Recuperado de https://bit.ly/3pidKrm

MINEDUC (2019). Minuta Docentes 2019. Centro de Estudios, Ministerio de Educación. Recuperado de https://bit.ly/3cgWAFc

MINEDUC (2020a, 27 marzo). Estudiantes Extranjeros. Ministerio de Educación. Recuperado de https://bit.ly/2SQYqWv

MINEDUC (2020b). Estudio Ministerio de Educación y Banco Mundial: Los estudiantes del país podrían perder hasta el $88 \%$ de los aprendizajes de un año. Recuperado de https://bit.ly/3ieiAUY

MINEDUC (2020c). Priorización Curricular Lenguaje y Comunicación / Lengua y Literatura. Santiago de Chile. Ministerio de Educación. Recuperado de: https://bit.ly/3pfJkFW

MINEDUC (2020d). Protocolo corona virus en establecimientos. Santiago, Chile: Ayuda Ministerio de Educación. Recuperado de https://bit.ly/3vQU4xc

Mishra, P. y Koehler, M. (2006). Technological pedagogical content knowledge: A framework for teacher knowledge. Teachers College Record, 108(6), 1017-1054. Recuperado de https://bit. Iy/2SMEDr3

Montoya, O., Gómez, M. y García, N. (2016). Estrategias para mejorar la comprensión lectora a través de las TIC. EDMETIC, 5(2), 71-93. Recuperado de https://bit.ly/3fLQrCR

Morales, K., Panes, R., Aguilera, H., Agurto, N. \& Bahamondes, M. y Olave, M. (2019). Acciones y percepciones de la gestión educativa en escuelas con alumnado migrante. Espacios 40(36) 20. https://bit.ly/2TEmFaL

Moreno, S. (2020). La innovación educativa en los tiempos del Coronavirus. Salutem Scientia Spiritus, (6), 14-26. Recuperado de https://bit.ly/3cdMwg8

Novoa, P. (2019). Estrategias de aplicación digital en la comprensión de textos narrativos. Investigaciones Sobre Lectura, 11, 37-55. https://doi.org/10.37132/isl.v0i11.277

Núñez, M. (s.f.). Materiales para un programa de comprensión lectora en la educación secundaria obligatoria. Recuperado de https://bit.ly/3fM4SXB 
OIT (2020). Sector rural y desarrollo local en América Latina y el Caribe (América Latina y el Caribe). Organización Internacional del Trabajo. Recuperado de https://bit.ly/3ifmH30.

Pedró, F. (2020). Covid-19 y educación superior en América latina y el Caribe: efectos, impactos y recomendaciones políticas. Fundación Carolina. Recuperado de https://bit.ly/3fHeByp

Pérez, A., Bermúdez, A. y Gallego, L. (2017). Fortalecimiento de la comprensión lectora en sus tres niveles literal, inferencial y crítico mediante el uso de la taxonomía de Barret (Tesis de magíster). Recuperado de https://bit.ly/3cwjtUZ

Propuestas Educación Mesa Social Covid-19. (2020). Didácticas para la proximidad: aprendiendo en tiempos de crisis. Recuperado de https://bit.ly/3yYvigs

Sistema de información de tendencias educativas en América Latina. (2020, 12 octubre). Sistematización de respuestas de los sistemas educativos de América Latina a la crisis de la COVID-19. Recuperado de https://bit.ly/2RXN2bj

VOA. (2015). División político administrativa [Mapa]. En Gobierno Regional del Maule y Programa Gestión Territorial para Zonas Rezagadas (2015). Atlas Territorial Región del Maule (p.43). Talca: Gobierno Regional de la Región del Maule.

\section{Anexos}

Carpeta en Google Drive con la encuesta y los resultados completos de la investigación: https://bit. ly/3fHMdfE

Código QR:

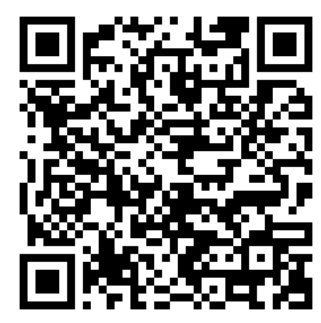

Cómo citar en APA este artículo:

Fierro, M., Morales, M., Norambuena, D., Bravo, B. y Contreras, P. (2021).

Objetivos priorizados del eje de lectura en la región del Maule, Chile:

problemáticas y desafíos. Revista Iberoamericana de Educación, 86(1), 135-152.

https://doi.org/10.35362/rie8614295 